\title{
Pulse propagation in decorated granular chains: An analytical approach
}

\author{
Upendra Harbola, ${ }^{1}$ Alexandre Rosas, ${ }^{2}$ Aldo H. Romero, ${ }^{3}$ Massimiliano Esposito, ${ }^{1,4}$ and Katja Lindenberg ${ }^{1}$ \\ ${ }^{1}$ Department of Chemistry and Biochemistry, and BioCircuits Institute, University of California-San Diego, La Jolla, \\ California 92093-0340, USA \\ ${ }^{2}$ Departamento de Física, CCEN, Universidade Federal da Paraíba, Caixa Postal 5008, 58059-900 João Pessoa, PB, Brazil \\ ${ }^{3}$ Cinvestav-Querétaro, Libramiento Norponiente 200, 76230 Fracc. Real de Juriquilla, Querétaro, Querétaro, Mexico \\ ${ }^{4}$ Center for Nonlinear Phenomena and Complex Systems, Université Libre de Bruxelles, Code Postal 231, Campus Plaine, \\ B-1050 Brussels, Belgium
}

(Received 15 September 2009; published 24 November 2009)

\begin{abstract}
We study pulse propagation in one-dimensional chains of spherical granules decorated with small grains placed between large granules. The effect of the small granules can be captured by replacing the decorated chains by undecorated chains of large granules of appropriately renormalized mass and effective interaction between the large granules. This allows us to obtain simple analytic expressions for the pulse propagation properties using a generalization of the binary collision approximation introduced in our earlier work [Phys. Rev. E 80, 031303 (2009); Phys. Rev. E 69, 037601 (2004)].
\end{abstract}

DOI: 10.1103/PhysRevE.80.051302

PACS number(s): 45.70. $-\mathrm{n}, 46.40 . \mathrm{Cd}, 43.25 .+\mathrm{y}, 05.65 .+\mathrm{b}$

\section{INTRODUCTION}

It is well known that an initial impulse applied at one end of a granular chain in the absence of gaps or precompression can result in solitary waves propagating through the medium $[1,2]$. Apart from addressing fundamental problems of pulse dynamics in the presence of highly nonlinear interactions, this scenario also has direct practical application, e.g., in designing shock absorbers $[3,4]$, sound scramblers $[5,6]$, and actuating devices [7]. Thus, in the recent past pulse propagation in one-dimensional (1D) chains of granules has been studied extensively both theoretically and experimentally [8-26].

Several configurations and parametrizations of the 1D chain have been used to study pulse propagation in the face of dissipation $[10,11,15,16]$ and of polydispersity in the structure and mass of the granules $[9,14,17,21-27]$. In particular, polydispersity is frequently introduced in a regular fashion such as in tapered chains (TCs), in which the size and/or mass of successive granules systematically decreases or increases [9,21-27]. Polydispersity is also introduced by distributing masses randomly $[28,29]$, by "decorating" chains with small masses placed regularly or randomly among larger masses [17,27], and by optimizing grain distribution [30] for particular purposes. In a recent study [17,27] it was shown that the shock absorbing properties of a granular chain can be enhanced quite dramatically by decorating the chain with smaller granules. To our knowledge, however, most studies on decorated chains have been numerical and hence restricted to specific parameter values. One exception is the early work of Nesterenko and Laizardi [31] based on a continuum approximation, which is difficult to implement for chains with rapid spatial variations such as are inherently present in decorated chains. It is clearly desirable to devise new theoretical means to obtain analytic results for these systems. This is the goal of the present work.

In a recent paper [32] we analyzed pulse propagation in a variety of undecorated TCs. Using a binary collision approximation, we presented analytical expressions for various quantities that characterize propagation of the pulse or pulse front in 1D TCs. However, an essential element of the success of this approximation is that a given pair of granules collides only once. This approach is therefore not directly applicable to decorated chains, where one (or more) small or light granule between two large/heavy ones will rattle back and forth. A similar conclusion about the inapplicability of a two-body collision approximation (different from ours) to decorated chains was also reached in Ref. [27]. On the other hand, the simplicity offered by the binary collision approximation is too tempting to discard it altogether. Instead, we have succeeded in extending this theoretical scheme to decorated chains.

Thus, in this work we present such a scheme that allows us to make use of the binary collision approximation for decorated chains in which single small or light granules are placed between large or heavy ones. The case of more than one small granule placed between large ones is not yet part of our scheme nor is it in most decorated configurations that have been considered (numerically) in the literature. Such a generalization is however entirely feasible. Our approach is based on a mapping of the decorated chain onto an effective undecorated chain. The effect of small decorating granules is included through a modified interaction between the bigger granules. This also requires the renormalization of the mass of the bigger granules. We present a systematic way to carry out this renormalization. The binary approximation is then used on the effective chain, which allows us to obtain analytical results for the quantities that characterize the pulse front propagation in these chains. We compare the analytical results with exact numerical results for two different decorated chains: simple decorated chains, where the sizes of the bigger particles are all the same, and decorated linear forward TCs, where the sizes of the bigger particles decrease linearly along the chain.

In Sec. II we introduce the general granular chain model in terms of rescaled (dimensionless) variables. In Sec. III we introduce decorated chains and present an effective description in terms of the renormalized interaction and masses. We then apply the binary collision approximation in Sec. IV and 
obtain analytical results for pulse properties. The analytic results are compared to numerical integration results in Sec. V. In Sec. VI we provide a final summary and some comments about ongoing work.

\section{MODEL}

We consider chains of granules all made of the same material of density $\rho$. When neighboring granules collide, they repel each other according to the power-law potential

$$
V=\frac{a}{n} r_{k}^{\prime}\left|y_{k}-y_{k+1}\right|^{n} \text {. }
$$

Here $y_{k}$ is the displacement of granule $k$ from its position at the beginning of the collision and $a$ is a constant determined by Young's modulus and Poisson's ratio [33,34]. The exponent $n$ is $5 / 2$ for spheres (Hertz potential) [34]. We have defined

$$
r_{k}^{\prime}=\left(\frac{2 R_{k}^{\prime} R_{k+1}^{\prime}}{R_{k}^{\prime}+R_{k+1}^{\prime}}\right)^{1 / 2}
$$

where $R_{k}^{\prime}$ is the principal radius of curvature of the surface of granule $k$ at the point of contact. When the granules do not overlap, the potential is zero. The equation of motion for the $k$ th granule is

$$
\begin{aligned}
M_{k} \frac{d^{2} y_{k}}{d \tau^{2}}= & \operatorname{ar}_{k-1}^{\prime}\left(y_{k-1}-y_{k}\right)^{n-1} \theta\left(y_{k-1}-y_{k}\right) \\
& -\operatorname{ar}_{k}^{\prime}\left(y_{k}-y_{k+1}\right)^{n-1} \theta\left(y_{k}-y_{k+1}\right),
\end{aligned}
$$

where $M_{k}=(4 / 3) \pi \rho\left(R_{k}^{\prime}\right)^{3}$. The Heaviside function $\theta(y)$ ensures that the elastic interaction between grains only exists if they are in contact. For the leftmost (rightmost) granule in the chain, only the second (first) term on the right-hand side of Eq. (3) is present. Initially the granules are placed along a line so that they just touch their neighbors in their equilibrium positions (no precompression), and all but the leftmost particle are at rest. The initial velocity of the leftmost particle $(k=1)$ is $V_{1}$ (the impulse). We define the dimensionless quantity

$$
\alpha \equiv\left[\frac{M_{1} V_{1}^{2}}{a\left(R_{1}^{\prime}\right)^{n+1 / 2}}\right]
$$

and the rescaled quantities $x_{k}, t, m_{k}$, and $R_{k}$ via the relations

$$
\begin{gathered}
y_{k}=R_{1}^{\prime} \alpha^{1 / n} x_{k}, \quad \tau=\frac{R_{1}^{\prime}}{V_{1}} \alpha^{1 / n} t, \\
R_{k}^{\prime}=R_{1}^{\prime} R_{k}, \quad M_{k}=M_{1} m_{k} .
\end{gathered}
$$

Equation (3) can then be rewritten as

$$
\begin{aligned}
m_{k} \ddot{x}_{k}= & r_{k-1}\left(x_{k-1}-x_{k}\right)^{n-1} \theta\left(x_{k-1}-x_{k}\right) \\
& -r_{k}\left(x_{k}-x_{k+1}\right)^{n-1} \theta\left(x_{k}-x_{k+1}\right),
\end{aligned}
$$

where a dot denotes a derivative with respect to $t$, and

$$
r_{k}=\left(\frac{2 R_{k} R_{k+1}}{R_{k}+R_{k+1}}\right)^{1 / 2} .
$$

The rescaled initial velocity is unity, i.e., $v_{1}(t=0)=1$. The velocity of the $k$ th granule in unscaled variables is simply $V_{1}$ times its velocity in the scaled variables.

\section{DECORATED CHAINS AND THE EFFECTIVE DYNAMICS}

There are several ways to construct a decorated chain. We shall consider decorated chains where small granules (all of the same size) alternate with larger granules. Our goal is to arrive at an analytically manageable effective description of a decorated chain. To be useful, such a description must be accurate in that it should capture (hopefully quantitatively) certain properties of the pulse dynamics. In particular, we attempt to capture the time dependence of the velocity profiles of the granules as the pulse moves along the chain, and the time that it takes a pulse to travel along the chain. We also try to characterize the frequency of oscillation of the small granules that separate each pair of large granules.

The frequency of oscillation of the small granules can be calculated by considering just a threesome of grains, two large ones with a small one in between. The analysis of a three-grain chain is also a convenient way to detail the mathematical analysis that is then repeated for a longer system. The three-grain chain is analyzed in Sec. III A. On the other hand, to characterize the large granules that will constitute the elements of our effective chain, the shortest unit that must be analyzed is one of five granules in the sequence large-small-large-small-large. This is the minimal chain that starts with a large granule (as do our long chains) and has an interior granule with small granules on either side (whose effects will be captured in the renormalized mass and renormalized interactions). The five-grain chain is analyzed in Sec. III B. Our effective chain of arbitrary length will then consist of all large granules, two end ones identified with the two end granules in the five-grain sequence, and any number of interior granules identified with the interior large granule in the five-granule sequence.

\section{A. Three-grain chain}

We first consider a chain of three granules labeled $k-1, k$, and $k+1$, granule $k$ being the small one. As noted above, this analysis will tell us something about the frequency of oscillation of the small granule, and it will also allow us to lay out the approximation scheme to be used in longer chains. The dynamics of the three granules is governed by the equations of motion

$$
\begin{aligned}
\ddot{x}_{k-1}= & -\mathcal{R}\left(x_{k-1}-x_{k}\right)^{n-1} \theta\left(x_{k-1}-x_{k}\right), \\
\ddot{x}_{k+1}= & \mathcal{R}\left(x_{k}-x_{k+1}\right)^{n-1} \theta\left(x_{k}-x_{k+1}\right), \\
m \ddot{x}_{k}= & \mathcal{R}\left(x_{k-1}-x_{k}\right)^{n-1} \theta\left(x_{k-1}-x_{k}\right) \\
& -\mathcal{R}\left(x_{k}-x_{k+1}\right)^{n-1} \theta\left(x_{k}-x_{k+1}\right) .
\end{aligned}
$$

To convey the method most clearly, we have here taken the 
masses $m_{k \pm 1}$ of the larger particles to be equal to each other and equal to unity in rescaled variables; $m$ is the mass of the small granule. The effective elastic constant $\mathcal{R}$ is given by Eq. (7) for a large granule of unit radius adjacent to a small one of radius $r$, that is,

$$
\mathcal{R}=\left(\frac{2 r}{1+r}\right)^{1 / 2} .
$$

The generalization to tapered chains is implemented later.

When granule $k-1$ is given an initial impulse, the energy is transported to granule $k$, which in turn transfers it to the last granule $k+1$. Since granule $k$ is small, it gets compressed between the two large granules and oscillates between them during the energy transfer process. The frequency of these oscillations can be expressed in terms of the maximum force that the granule $k$ experiences from granule $k+1$ or $k-1$. We follow Melo et al. [35] and write

$$
x_{k}(t)=\bar{x}_{k}(t)+A \sin (\omega t+\phi) .
$$

Here $A, \omega$, and $\phi$ depend on the size ratio between the small and large granules and may also depend on the time. We assume that these time dependences are negligibly weak during the time of energy transfer from $k-1$ to $k+1$. The first term $\bar{x}_{k}$ represents the average motion around which the particle executes oscillations of small amplitude $A$ with frequency $\omega$. Substituting Eq. (10) into Eq. (8c) and assuming that the amplitude $A$ is much smaller than $\bar{x}_{k}-x_{k+1}$ and $x_{k-1}$ $-\bar{x}_{k}$, we expand in $A$ and from the lowest two orders we obtain

$$
\begin{aligned}
m \ddot{\bar{x}}_{k} \approx & \mathcal{R}\left(x_{k-1}-\bar{x}_{k}\right)^{n-1} \theta\left(x_{k-1}-\bar{x}_{k}\right) \\
& -\mathcal{R}\left(\bar{x}_{k}-x_{k+1}\right)^{n-1} \theta\left(\bar{x}_{k}-x_{k+1}\right), \\
m \omega^{2} \approx & \mathcal{R}(n-1)\left[\left(x_{k-1}-\bar{x}_{k}\right)^{n-2} \theta\left(x_{k-1}-\bar{x}_{k}\right)\right. \\
+ & \left.\left(\bar{x}_{k}-x_{k+1}\right)^{n-2} \theta\left(\bar{x}_{k}-x_{k+1}\right)\right] .
\end{aligned}
$$

In the following, for economy of notation we do not write the $\theta$ functions explicitly, but their presence restricting the interactions to compressions should be kept in mind.

The small granule oscillates with maximum frequency around the "equilibrium" position, defined as the position at which the forces $\bar{F}$ obtained using the average displacement of the smaller granule, cancel each other on the two sides, i.e., where $\ddot{\bar{x}}_{k}=0$. Setting

$$
\bar{F}=\mathcal{R}\left(x_{k-1}-\bar{x}_{k}\right)^{n-1}=\mathcal{R}\left(\bar{x}_{k}-x_{k+1}\right)^{n-1}
$$

[cf. Eq. (11a)], and using Eq. (11b), we can write for the maximum frequency $f=\omega / 2 \pi$,

$$
f \approx \frac{1}{2 \pi} \sqrt{\frac{2(n-1)}{m}} \mathcal{R}^{1 / 2(n-1)} \bar{F}^{(n-2) / 2(n-1)} .
$$

This is the theoretical prediction for the frequency of the small granule that we will later compare with numerical simulation results once we are able to calculate the value of the average force $\bar{F}$ using our binary collision approximation.

At the time when the small particle executes oscillations with maximum frequency, we have $\ddot{\bar{x}}_{k}=0$. From Eq. (11a), this implies that

$$
\bar{x}_{k}=\frac{1}{2}\left(x_{k+1}+x_{k-1}\right) \text {. }
$$

Also, note that from Eqs. (8a)-(8c)

$$
m \ddot{x}_{k}+\ddot{x}_{k-1}+\ddot{x}_{k+1}=0 .
$$

Equation (15) is the statement that the net force on the system is zero. Substituting Eq. (10) into Eq. (15), using Eq. (14), and ignoring the small oscillatory part, we get $\left(\frac{m}{2}\right.$ $+1)\left(\ddot{x}_{k-1}+\ddot{x}_{k+1}\right)=0$, which describes a system of two granules each with a renormalized mass

$$
\mu=1+\frac{m}{2} .
$$

Replacing $x_{k}$ in Eqs. (8a) and (8b) by the form assumed in Eq. (10) and using Eq. (14) we obtain

$$
\begin{aligned}
& \mu \ddot{x}_{k-1} \approx-\frac{\mathcal{R}}{2^{n-1}}\left(x_{k-1}-x_{k+1}\right)^{n-1}+\frac{m A}{2} \omega^{2} \sin (\omega t+\phi), \\
& \mu \ddot{x}_{k+1} \approx \frac{\mathcal{R}}{2^{n-1}}\left(x_{k-1}-x_{k+1}\right)^{n-1}-\frac{m A}{2} \omega^{2} \sin (\omega t+\phi) .
\end{aligned}
$$

Since the oscillation amplitude $A$ is small, we neglect the oscillatory terms in Eq. (17) and simply write

$$
\begin{gathered}
\mu \ddot{x}_{k-1} \approx-\frac{\mathcal{R}}{2^{n-1}}\left(x_{k-1}-x_{k+1}\right)^{n-1}, \\
\mu \ddot{x}_{k+1} \approx \frac{\mathcal{R}}{2^{n-1}}\left(x_{k-1}-x_{k+1}\right)^{n-1} .
\end{gathered}
$$

Equations (18) define the effective interaction potential $V_{\text {eff }}$ between granules $k-1$ and $k+1$. Thus we can treat the system of two identical granules decorated with a smaller granule as an effective system of two granules (now relabeled as $k$ and $k+1$ ) with effective masses $\mu$ interacting with an effective potential given by

$$
V_{e f f}=\frac{\mathcal{R}}{n 2^{n-1}}\left(x_{k}-x_{k+1}\right)^{n} .
$$

Thus the overall effect of an intermediate granule is to reduce the interaction strength between granules $k-1$ and $k$ +1 (in the original chain) and increase their masses. This shows that the pulse will be wider as compared to the monodisperse case. Result (19) remains valid as long as the oscillation amplitude of the small granule is small enough so that only the lowest order terms in the expansion [Eqs. (11a) and (11b)] are important. We shall see that this puts an upper limit on the size of the smaller granule. 


\section{B. Long-decorated chain}

Using the three-grain chain to study a tapered chain leads to a certain ambiguity because when there are two large granules of distinct masses around a small one, the renormalized masses will be different depending on the pairing. Thus, the renormalized mass of the $k$ th granule would have different values when considering the interaction between granules $k-1$ and $k$ or between $k$ and $k+1$. This ambiguity can be suppressed if we consider a chain of five granules centered on granule $k$, labeled from $k-2$ to $k+2$. Granules $k-1$ and $k+1$ are small ones of radius $r$. The radius of large granule $i=k, k \pm 2$ is $R_{i}>r$. Moreover, this approach is useful even for a uniform decorated chain because it naturally shows the distinction between a granule inside the chain and a granule on either border. The dynamics of this chain of granules is governed by the set of equations

$$
\begin{gathered}
m_{k-2} \ddot{x}_{k-2}=-r_{k-2}\left(x_{k-2}-x_{k-1}\right)^{n-1} \theta\left(x_{k-2}-x_{k-1}\right), \\
m_{i} \ddot{x}_{i}=r_{i-1}\left(x_{i-1}-x_{i}\right)^{n-1} \theta\left(x_{i-1}-x_{i}\right) \\
\quad-r_{i}\left(x_{i}-x_{i+1}\right)^{n-1} \theta\left(x_{i}-x_{i+1}\right), \quad i=k, k \pm 1, \\
m_{k+2} \ddot{x}_{k+2}=r_{k+1}\left(x_{k+1}-x_{k+2}\right)^{n-1} \theta\left(x_{k+1}-x_{k+2}\right) .
\end{gathered}
$$

For the smaller granules, as was done in Eq. (10), we again assume a separation into an average displacement and an oscillatory contribution about the average. Following the same steps that led from Eq. (10) to Eq. (14), we now obtain for the smaller granules

$$
\bar{x}_{i}=\frac{x_{i-1}+\alpha_{i} x_{i+1}}{1+\alpha_{i}},
$$

where $i=k-1, k+1$ and

$$
\alpha_{i}=\left(\frac{r_{i}}{r_{i-1}}\right)^{1 /(n-1)} .
$$

Using Eq. (21) in Eq. (20), the pulse propagation in a decorated chain of five granules can be described by pulse propagation along an effective chain of three large granules, the "left" $(l)$, the "middle" $(m)$, and the "right" $(r)$ of renormalized masses

$$
\begin{gathered}
\mu_{m}=m_{k}+\frac{m}{1+\left(\frac{r_{k-2}}{r_{k-1}}\right)^{1 /(n-1)}}+\frac{m}{1+\left(\frac{r_{k+1}}{r_{k}}\right)^{1 /(n-1)}}, \\
\mu_{l}=m_{k-2}+\frac{m}{1+\left(\frac{r_{k-1}}{r_{k-2}}\right)^{1 /(n-1)}}, \\
\mu_{r}=m_{k+2}+\frac{m}{1+\left(\frac{r_{k}}{r_{k+1}}\right)^{1 /(n-1)}} .
\end{gathered}
$$

Henceforth we relabel the three grains in the effective chain as $(l, m, r) \rightarrow(k-1, k, k+1)$. The effective interaction between the $k$ th and $(k+1)$ st granules (relabeled) is given by

$$
V_{e f f}=\frac{\zeta_{k}(n)}{n}\left(x_{k}-x_{k+1}\right)^{n}
$$

where

$$
\zeta_{k}(n)=\frac{1}{\left[\left(\frac{1}{r_{k}}\right)^{1 /(n-1)}+\left(\frac{1}{r_{k+1}}\right)^{1 /(n-1)}\right]^{n-1}},
$$

and where $r_{k}$ is now given by

$$
r_{k}=\sqrt{\frac{2 R_{k} r}{R_{k}+r}} .
$$

A long decorated chain can now be represented by an effective chain of large granules where the mass of the $k$ th granule (except for those at the edges of the chain) is $\mu_{m}$. Note that the effective mass $\mu_{m}$ is modified from $m_{k}$ by the presence of the two small granules on either side of it in the decorated chain. The masses of the granules at the edges of the long effective chain are $\mu_{l}$ and $\mu_{r}$ and are modified from $m_{k \mp 2}$ by only a single small mass. The interaction between the $k$ th and $(k+1)$ st (relabeled) granules in the effective long chain is given by Eq. (24) with Eq. (25). Hence, as in the three-grain chain, the interactions are weaker and the masses larger in the effective chain than in the original decorated chain. For the special case $R_{k}=R_{k \pm 2}$ in the five-granule decorated chain, Eqs. (21) and (24) reduce to Eqs. (14) and (19), respectively.

\section{BINARY COLLISION APPROXIMATION FOR EFFECTIVE CHAIN}

We now use the binary collision approximation to obtain analytic expressions for the pulse propagating along a decorated chain and make the connection with the effective description of Sec. III. The following analysis is based on Ref. [32].

In the binary collision approximation we assume that the transfer of energy along the chain occurs via a succession of two-particle collisions. First, particle $k=1$ with velocity $v_{1}$ $=1$ collides with initially stationary particle $k=2$, which then acquires a velocity $v_{2}$ and collides with stationary particle $k=3$, and so on. Using energy and momentum conservation and remembering that in the effective chain the granules have effective mass $\mu_{k}$, it can easily be shown that the velocity $v_{k}$ of the $k$ th granule is given by

$$
v_{k}=\prod_{k^{\prime}=1}^{k-1} \frac{2}{1+\frac{\mu_{k^{\prime}+1}}{\mu_{k^{\prime}}}} \text {. }
$$

The coupled dynamics of any two interacting granules in our models can be reduced to a single-particle dynamics. This is helpful toward obtaining analytical expressions for some of the pulse properties, as we shall see below. For this purpose, we pick granules $k$ and $k+1$ and introduce the difference variable

$$
z_{k}=x_{k}-x_{k+1} .
$$

The equation of motion for the difference variable is obtained by subtracting the equations of motion of the two granules during a collision, 


$$
\begin{gathered}
\ddot{x}_{k}=-\frac{\zeta_{k}(n)}{\mu_{k}}\left(x_{k}-x_{k+1}\right)^{n-1}, \\
\ddot{x}_{k+1}=\frac{\zeta_{k}(n)}{\mu_{k+1}}\left(x_{k}-x_{k+1}\right)^{n-1},
\end{gathered}
$$

where $\zeta_{k}(n)$ is defined in Eq. (25). Equation (29) with Eq. (28) immediately leads to

$$
\ddot{z}_{k}=-\frac{\zeta_{k}(n)}{\mathcal{M}_{k}} z_{k}^{n-1}
$$

where

$$
\mathcal{M}_{k}=\frac{\mu_{k} \mu_{k+1}}{\mu_{k}+\mu_{k+1}}
$$

is the reduced mass of the coupled system.

Equation (30) is the equation of motion of a particle of effective mass $\mathcal{M}_{k}$ in the potential $\zeta_{k}(n) z_{k}^{n} / n$ defined for $z_{k}$ $\geq 0$. The initial conditions are $\dot{z}_{k}(0)=v_{k}$ since the velocity of granule $k+1$ is zero before the collision, and $z_{k}(0)=0$ since there is no precompression. The energy conservation condition

$$
\frac{1}{2} \dot{z}_{k}^{2}(t)+\frac{\zeta_{k}(n)}{n \mathcal{M}_{k}} z_{k}^{n}(t)=\frac{1}{2} \dot{z}_{k}^{2}(0)
$$

leads to

$$
\dot{z}_{k}(t)=\left(\dot{z}_{k}^{2}(0)-\frac{2 \zeta_{k}(n)}{n \mathcal{M}_{k}} z_{k}^{n}(t)\right)^{1 / 2} .
$$

We say that the pulse arrives at granule $k$ when the velocity of granule $k$ surpasses that of granule $k-1$ and that it moves on to granule $k+1$ when the velocity of the $(k+1)$ st granule surpasses that of the $k$ th granule. The residence time $T_{k}$ on granule $k$ is the time that granule $k$ takes to transfer the pulse from $k-1$ to $k+1$ and is given by

$$
T_{k}=\int_{0}^{z_{k}^{\max }} \frac{d z_{k}}{z_{k}}=\int_{0}^{z_{k}^{\max }} \frac{d z_{k}}{\left(\dot{z}_{k}^{2}(0)-\frac{2 \zeta_{k}(n)}{n \mathcal{M}_{k}} z_{k}^{n}(t)\right)^{1 / 2}},
$$

where $z_{k}^{\max }$ is the compression when the velocities of particles $k$ and $k+1$ are equal (which is also the maximum compression),

$$
z_{k}^{\max }=\left(\frac{n \mathcal{M}_{k}}{2 \zeta_{k}(n)} \dot{z}_{k}^{2}(0)\right)^{1 / n} .
$$

The integral can be performed exactly to yield

$$
T_{k}=\sqrt{\pi}\left(\frac{n \mathcal{M}_{k}}{2 \zeta_{k}(n)}\right)^{1 / n}\left[\dot{z}_{k}(0)\right]^{2 / n-1} \frac{\Gamma(1+1 / n)}{\Gamma(1 / n+1 / 2)} .
$$

Finally, the total time taken by the pulse to pass the $k$ th granule is obtained by summing $T_{k}$,

$$
t=\sum_{k^{\prime}=1}^{k} T_{k^{\prime}}
$$

In the next section we compare these various quantities obtained from the binary collision approximation implemented

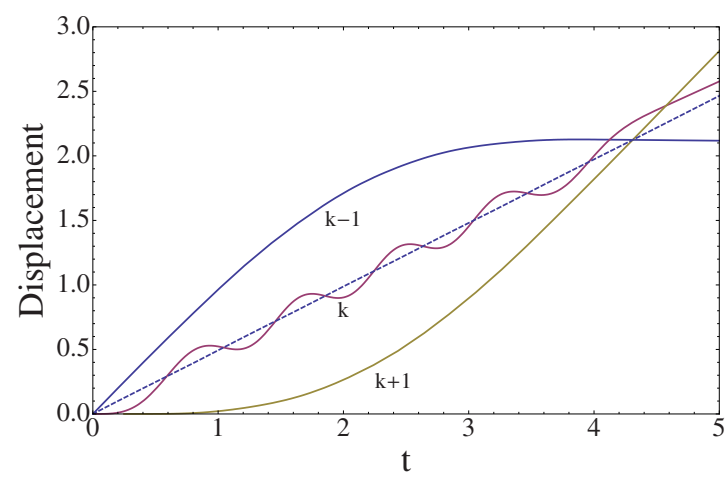

FIG. 1. (Color online) Solid lines: displacements of the three granules in a three-granule chain as a function of time. Dashed line: average displacement of the small middle granule.

on the effective undecorated chains with decorated chain numerical integration results.

At this point we can also calculate the average force that appears in Eq. (13). When the two granules $k$ and $k+1$ collide, the maximum force between them corresponds to the maximum compression $z_{k}^{\max }$. Thus the maximum force is

$$
\bar{F}=\zeta_{k}(n)\left(z_{k}^{\max }\right)^{n-1}=\left[\zeta_{k}(n)\right]^{1 / n}\left(\frac{n \mathcal{M}_{k} \dot{z}_{k}^{2}(0)}{2}\right)^{(n-1) / n} .
$$

The explicit formulas for the three-grain configuration considered in Sec. III A correspond to using Eq. (31) with $\mu_{k}$ $=\mu_{k+1}=\mu$ and hence $\mathcal{M}_{k}=\mu / 2, \zeta_{k}(n)=\mathcal{R} / 2^{n-1}$, and $\dot{z}_{k}^{2}(0)$ $=1$. We obtain

$$
\bar{F}=\frac{n \mu}{4}\left(\frac{\mathcal{R}}{n 2^{n-3} \mu}\right)^{1 / n},
$$

where $\mathcal{R}$ and $\mu$ are given in Eqs. (9) and (16), respectively. Substituting this in Eq. (13), we obtain

$$
f \approx \frac{1}{2 \pi} \sqrt{\frac{2(n-1) \mu}{m}}\left(\frac{\mathcal{R}}{\mu}\right)^{1 / n}\left(\frac{n}{8}\right)^{(n-2) / 2 n} .
$$

In the next section we compare this frequency with that obtained from the numerical solution of the exact dynamical equations, Eqs. (8a)-(8c).

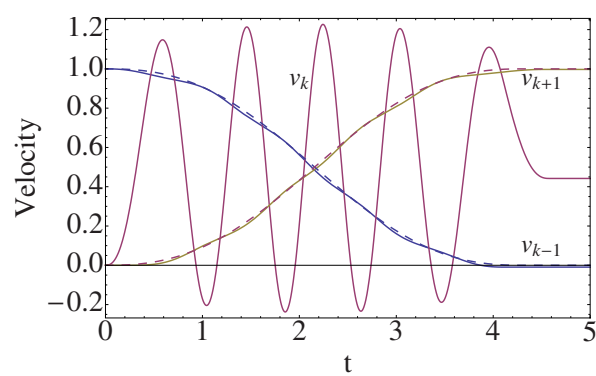

FIG. 2. (Color online) Typical velocity profiles of three granules (continuous curves) obtained from the numerical solution of the exact dynamical equations. The small particle of radius $r=0.3$ oscillates with almost constant frequency while the amplitude of oscillations shows a weak time dependence. The dashed curves represent results from the effective dynamics. 


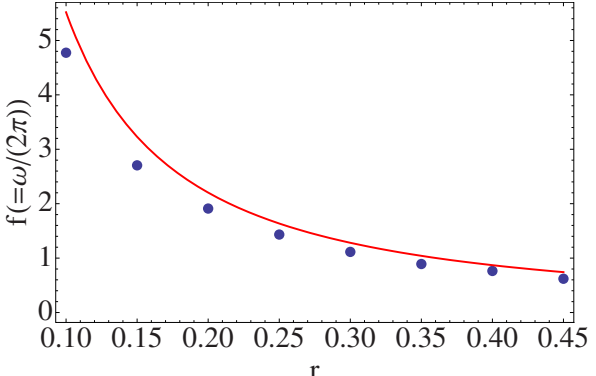

FIG. 3. (Color online) The dots represent the numerical results for the average angular frequency of oscillations of the small particle during the time of energy transfer from granule $k-1$ to $k+1$. The solid curve is the theoretical estimate based on the binary collision approximation using the effective interaction.

\section{COMPARISON WITH NUMERICAL RESULTS}

While our binary collision approximation is valid for any $n>2$ [12], in our numerical comparisons we focus on spherical granules. We start with the chain of three particles of Sec. III A. Our first figure, Fig. 1, shows that the important step of neglecting oscillatory terms as detailed in the derivation of Eqs. (11a), (11b), and (18) is justified. The figure shows the displacements of the three granules as a function of time. The dashed curve shows the average displacement [Eq. (14)] of the small granule. The "small amplitude" in question is the difference between the oscillatory curve and the dashed curve compared to the differences between the dashed curve and the two large-granule displacement curves.

Figure 2 shows the velocity profile of the granules obtained from the solution of the exact dynamical equations for the three granules. The small granule oscillates a number of times before the chain disintegrates. In the same figure we also show a comparison with the results obtained from the effective dynamical equations, Eqs. (18).

As we see from the figure, the behavior of the large particles is captured very accurately by the effective interaction given by Eq. (19). We observe this quality of agreement as long as the radius of the small granule is less than about $40 \%$ of the larger ones. We return to this point later in the paper. The effective description improves as the size of the small granule decreases.

Although it is not necessary for the characterization of the propagation of the pulse, it is interesting to compare the theoretical [Eq. (40)] and numerical results for the oscillation frequency of the small particle as a function of its radius. The theoretical result is shown as a solid curve in Fig. 3. The numerical results are obtained by fitting the derivative of Eq. (10), $v(t)=0.5+\omega A \cos (\omega t+\phi)$ (the particle velocity oscillates around 0.5$)$. The resulting frequencies are shown as dots in the figure. While the theoretical curve appears closer to the numerical results for the larger granules, the percent

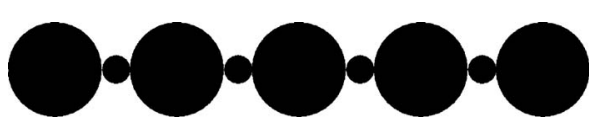

FIG. 4. Schematic of a simple decorated chain of nine granules.

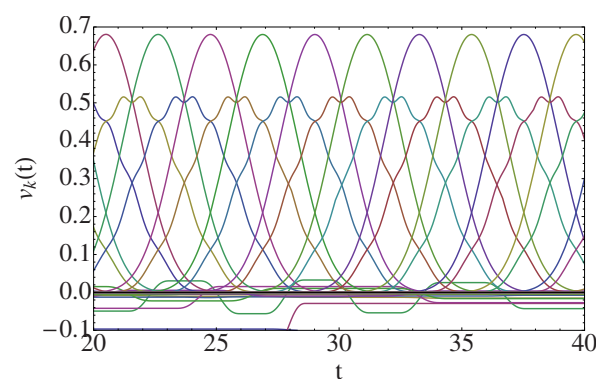

FIG. 5. (Color online) Velocity profile of granules in a simple decorated chain with small granules of radii $r=0.3$.

difference between the two is $14 \%$ for the smallest granules and $20 \%$ for the largest granules shown in the figure.

\section{A. Simple decorated chain}

A simple decorated chain is one in which the large granules all have the same radius. For our numerical simulations of a simple decorated chain we consider $N=50$ large granules, with each pair of large granules separated by a small granule. Initially all the granules are placed such that they barely touch their nearest neighbors (no precompression). In all decorated chains, we consider the first granule to be a large one. A schematic of a typical simple decorated chain is shown in Fig. 4.

Figure 5 shows the velocity pulse propagating through the chain. The smooth higher velocity peaks are associated with the large granules, while the lower peaks with oscillatory contributions are those of the small granules. The oscillations in the velocity profiles of the small particles are most pronounced around their maximum velocity. This is due to the fact that the forces on the two sides of the small granule tend to cancel each other around that time.

Almost all the noisy behavior at the bottom of Fig. 5 is due to the oscillations of the smaller granules. None of these oscillatory and noisy contributions affect the smoothness of the pulse front located on the larger granules. We also note that the initial energy given to the left edge of the chain is transported mainly through the larger granules. The energy of the small granules is negligible compared to that of the larger ones.

In Fig. 6 we compare the exact dynamics of the larger

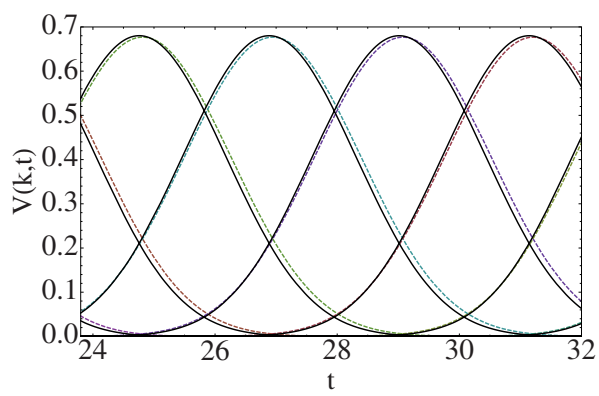

FIG. 6. (Color online) Exact results (solid curves) for the velocity profiles of the larger granules in a simple decorated chain for $r=0.3$. The dotted curves show results from the solution of the effective dynamical equations. 


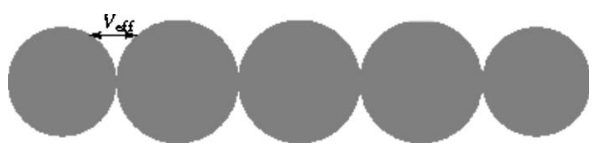

FIG. 7. Schematic of the effective undecorated chain associated with Fig. 4. Each pair of granules interacts with the same pair potential $V_{\text {eff }}$ but the end masses are smaller than the interior masses.

granules with the results obtained from the solution of the dynamical equations of the associated effective undecorated chain. We show only a small stretch in the figure, but the agreement is typical of the entire chain. A schematic of this chain is shown in Fig. 7. The dynamical equations for the two edges of the effective chain are given in Eq. (18) with the associated effective mass [Eq. (16)], or equivalently, the effective mass [Eq. (23b) or Eq. (23c)] for equal large granules. The interior masses satisfy the dynamical equation (with $\theta$ functions omitted)

$$
\ddot{x}_{k}=\frac{\mathcal{R}}{2^{n-1} \mu}\left[\left(x_{k-1}-x_{k}\right)^{n-1}-\left(x_{k}-x_{k-1}\right)^{n-1}\right]
$$

with the effective mass [Eq. (23a)], which for the simple decorated chain reduces to

$$
\mu_{k}=\mu=1+m .
$$

For clarity, in Fig. 6 we only show a section of the chain between $k=12$ and $k=15$ (here $k$ counts only the larger granules). The results from the effective dynamics are in excellent agreement with the exact solution.

For a simple decorated chain, $T_{k}$ is independent of $k$ (except for the edge granules, which have a different effective mass) and is given by

$$
T_{k}=\sqrt{\pi}\left(\frac{n 2^{n-3} \mu}{\mathcal{R}^{n-1}}\right)^{1 / n} \frac{\Gamma(1+1 / n)}{\Gamma(1 / n+1 / 2)},
$$

with the effective mass of Eq. (42). The time $t$ defined in Eq. (37) therefore varies linearly with $k$. The slope of the line is determined by $T_{k}$. In Fig. 8 we show numerical results for the

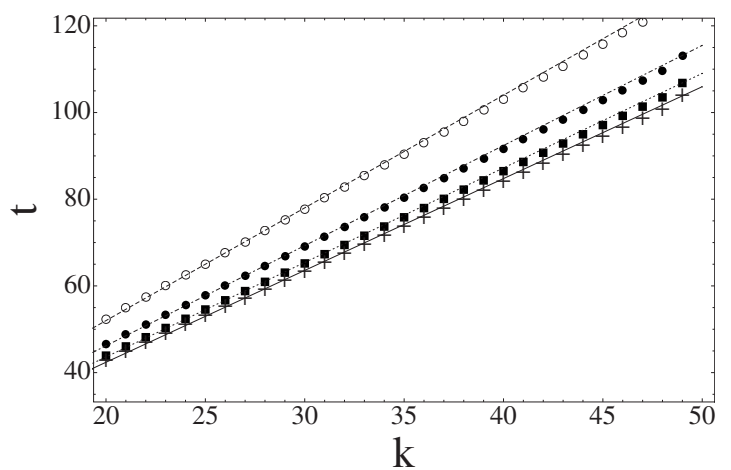

FIG. 8. Time $t$ taken by the pulse to reach the $k$ th granule in a simple decorated chain for $r=0.1$ (open circles), $r=0.2$ (filled circles), $r=0.3$ (squares), and $r=0.4$ (plus signs). The theoretical results are shown with dashed $(r=0.1)$, dashed-dotted $(r=0.2)$, dotted $(r=0.3)$, and continuous $(r=0.4)$ lines.

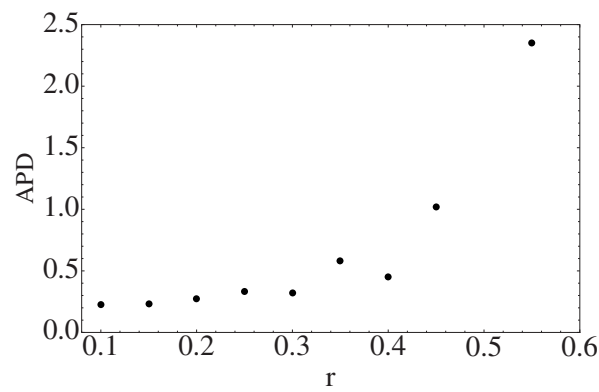

FIG. 9. Average percent difference (APD) between the exact dynamics and the effective dynamics as a function of the radius of the small granules. The APD is calculated by taking the percent difference in the pulse amplitude for each granule in the chain, summing over all granules, and dividing by the total number of granules.

time taken by the pulse to reach the $k$ th granule.

Note that $k$ here labels only the larger particles in the actual chain, and for clarity we show results for the restricted range $20<k<50$. Various symbols represent different values of $r$. In the same figure we also show the analytical result Eq. (37) of the binary collision approximation. No fitting parameters are involved, and the agreement of the two results is again gratifying. This shows that the effective binary theory can give quantitatively reliable results for simple decorated chains.

These quantitatively reliable results are obtained provided the radius of the small granules is no larger than about $r$ $\sim 0.4$. In Fig. 9 we show the average percent difference in the pulse amplitude calculated from the decorated dynamics and from the effective undecorated one as a function of the radius of the small granules. The average percent difference is calculated by taking the percent difference in the pulse amplitude for each granule in the chain, summing over all granules, and dividing by the total number of granules. The average percent difference remains under $1 \%$ up to about $r$ $=0.4$ and beyond that rises sharply.

\section{B. Tapered-decorated chain}

We next consider a linearly tapered chain decorated with small granules of the same size. For numerical purposes, we again consider a tapered chain of $N=50$ larger granules. The radius of the $k$ th large granule is $R_{k}=1-S(k-1)$ and $S$ is the tapering parameter [32]. This chain is then decorated with the smaller granules of radius $r$. A schematic of a typical tapered decorated chain is shown in Fig. 10.

Our effective description is valid as long as the amplitudes of oscillations of the smaller particles are small compared to their average displacement. This occurs as long as the size of the small granules is roughly less than $40 \%$ of the

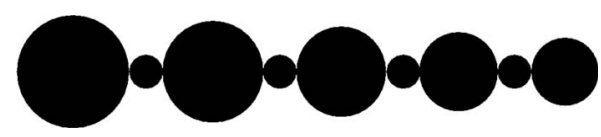

FIG. 10. Schematic of a tapered-decorated chain of nine granules. 


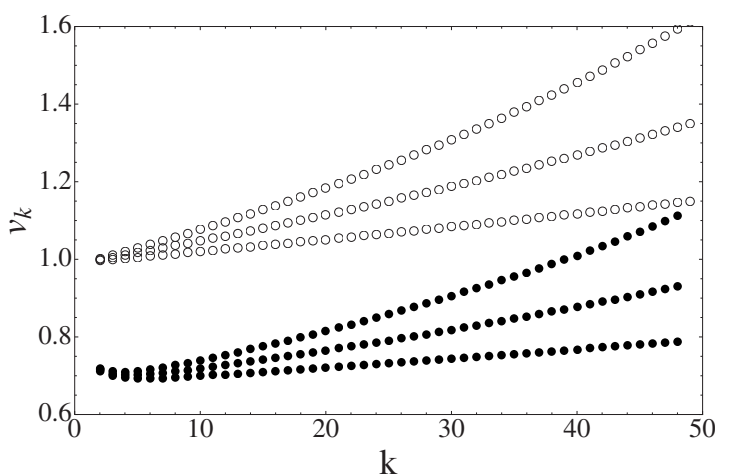

FIG. 11. Change in the pulse amplitude as a function of $k$. The filled and the empty circles represent the exact numerical result and the binary collision approximation result, respectively. The three different data sets are for $S=0.002,0.004,0.006$ from bottom to top. In all cases $r=0.3$.

size of the larger granules. The value of $S$ is therefore restricted by the length of the tapered chain. This is the reason for choosing linear rather than geometric tapering for this analysis [32], which is even more restrictive.

In Fig. 11 we show the change in the pulse amplitude with the granule number. As already noted in the case of undecorated tapered chains [32], the absolute value of the pulse amplitude is not captured correctly by the binary approximation, but the rate of change of the amplitude is close to that obtained from the exact results.

However, other characteristics of the pulse are extremely well captured by the binary approximation. We next compare the binary prediction for the time spent by each granule in the pulse. The analytical result for the residence time of the pulse on granule $k$ is given by $T_{k}$ in Eq. (34). In Fig. 12 we compare the results obtained from the exact dynamics to those of the binary collision approximation for $r=0.1,0.2$, and 0.3. Excellent agreement is again obtained between the two results. The small initial disagreement is due to an edge effect. The residence time of the pulse decreases rapidly as the pulse propagates through the chain, as it does in a nondecorated forward tapered chain [32]. Note that the residence time of the pulse on granule $k$ decreases with increasing $r$ because the increase in the effective mass is accompanied by an even greater increase in the effective interaction between granules. In the inset of Fig. 12, we show the results for $r$ $=0.4$. Here the exact and approximate results disagree for large $k$ values because the size difference between the larger and the smaller granules decreases to where the description in terms of an effective chain breaks down.

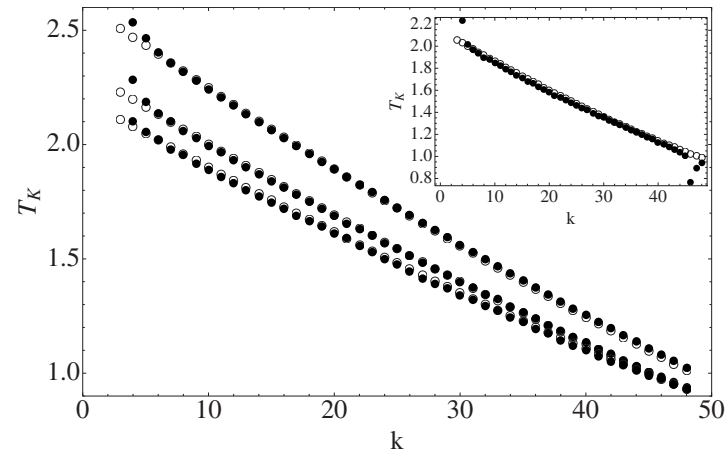

FIG. 12. Residence time $T_{k}$ of the pulse on the $k$ th granule for $r=0.1,0.2$, and 0.3 from top to bottom, and $S=0.01$. Numerical and analytical results are represented by the filled and open circles, respectively. The inset shows results for $r=0.4$.

\section{CONCLUSIONS}

We have studied pulse propagation in decorated chains, that is, chains in which small and large granules alternate. We have presented a method to map the actual chain into an effective undecorated chain. This effective description allows us to obtain analytical results using the binary collision approximation for quantities that characterize the pulse properties in decorated chains. The effective description is extremely accurate as long as the size ratio of the small granules to the large granules is less than $\sim 0.40$. We have explicitly considered two different chains, simple decorated chains and forward-tapered decorated chains. For simplicity, in order to obtain an effective description, we have assumed a fixed size for the small granules. However the method can easily be applied with little modifications to other more complicated decorated chains where small granules are also allowed to vary in size. The method can also be implemented in decorated chains with more than one large granule between the small ones. A more extensive generalization is necessary to deal with decorated chains with more than one small granule between large ones and thence to other size profiles. This work is in progress.

\section{ACKNOWLEDGMENTS}

Acknowledgment is made to the Donors of the American Chemical Society Petroleum Research Fund for partial support of this research (K.L. and U.H.). A.R. acknowledges support from Pronex-CNPq-FAPESQ and CNPq. M.E. was supported by the FNRS Belgium (chargé de recherches) and by the government of Luxembourg (Bourse de formation recherches). A.H.R. acknowledges support by CONACyT Mexico under Projects No. J-59853-F and No. J-83247-F.
[1] V. F. Nesterenko, J. Appl. Mech. Tech. Phys. 24, 733 (1983).

[2] A. N. Lazaridi and V. F. Nesterenko, J. Appl. Mech. Tech. Phys. 26, 405 (1985).

[3] C. Daraio, V. F. Nesterenko, E. B. Herbold, and S. Jin, Phys. Rev. Lett. 96, 058002 (2006).

[4] J. Hong, Phys. Rev. Lett. 94, 108001 (2005).
[5] V. F. Nesterenko, C. Daraio, E. B. Herbold, and S. Jin, Phys. Rev. Lett. 95, 158702 (2005).

[6] C. Daraio, V. F. Nesterenko, E. B. Herbold, and S. Jin, Phys. Rev. E 72, 016603 (2005).

[7] D. Khatri, C. Daraio, and P. Rizzo, Proc. SPIE 6934, 69340U (2008). 
[8] V. F. Nesterenko, Dynamics of Heterogeneous Materials (Springer, New York, 2001).

[9] M. Nakagawa, J. H. Agui, D. T. Wu, and D. V. Extramiana, Granular Matter 4, 167 (2003).

[10] S. Job, F. Melo, A. Sokolow, and S. Sen, Phys. Rev. Lett. 94, 178002 (2005).

[11] A. Rosas and K. Lindenberg, Phys. Rev. E 68, 041304 (2003).

[12] A. Rosas and K. Lindenberg, Phys. Rev. E 69, 037601 (2004).

[13] E. J. Hinch and S. Saint-Jean, Proc. R. Soc. London, Ser. A 455, 3201 (1999).

[14] P. J. Wang, J. H. Xia, Y. D. Li, and C. S. Liu, Phys. Rev. E 76, 041305 (2007).

[15] A. Rosas, A. H. Romero, V. F. Nesterenko, and K. Lindenberg, Phys. Rev. Lett. 98, 164301 (2007).

[16] A. Rosas, A. H. Romero, V. F. Nesterenko, and K. Lindenberg, Phys. Rev. E 78, 051303 (2008).

[17] S. Sen, Phys. Rep. 462, 21 (2008).

[18] C. Coste, E. Falcon, and S. Fauve, Phys. Rev. E 56, 6104 (1997).

[19] V. F. Nesterenko, J. Phys. IV 4, C8-729 (1994).

[20] A. Sokolow, E. G. Bittle, and S. Sen, EPL 77, 24002 (2007).

[21] D. T. Wu, Physica A 315, 194 (2002).

[22] R. L. Doney and S. Sen, Phys. Rev. E 72, 041304 (2005).
[23] A. Sokolow, J. M. M. Pfannes, R. L. Doney, M. Nakagawa, J. H. Agui, and S. Sen, Appl. Phys. Lett. 87, 254104 (2005).

[24] S. Sen, F. S. Manciu, and M. Manciu, Physica A 299, 551 (2001).

[25] F. Melo, S. Job, F. Santibanez, and F. Tapia, Phys. Rev. E 73, 041305 (2006).

[26] S. Job, F. Melo, A. Sokolow, and S. Sen, Granular Matter 10, 13 (2007).

[27] R. Doney and S. Sen, Phys. Rev. Lett. 97, 155502 (2006).

[28] A. Sokolow and S. Sen, Ann. Phys. 322, 2104 (2007).

[29] A.-L. Chen and Y.-S. Wang, Physics B 392, 369 (2007).

[30] F. Fraternali, M. A. Porter, and C. Daraio, Mech. Adv. Mater. Structures 16, 8 (2009).

[31] V. F. Nesterenko and A. N. Lazaridi, in Impulse Treatment of Materials (Russian, Novosibirsk, 1990), pp. 30-42.

[32] U. Harbola, A. Rosas, M. Esposito, and K. Lindenberg, Phys. Rev. E 80, 031303 (2009).

[33] L. D. Landau and E. M. Lifshitz, Theory of Elasticity (Addison-Wesley, Massachusetts, 1959).

[34] H. Hertz, J. Reine Angew. Math. 92, 156 (1881).

[35] S. Job, F. Santibanez, F. Tapia and F. Melo, Phys. Rev. E 80, 025602(R) (2009). 